\title{
Modeling Broadcasting in IEEE 802.11p/WAVE Vehicular Networks
}

\author{
Claudia Campolo, Alexey Vinel, Antonella Molinaro, and Yevgeni Koucheryavy
}

\begin{abstract}
IEEE 802.11p/WAVE (Wireless Access in Vehicular Environments) is an emerging family of standards intended to support wireless access in Vehicular Ad Hoc Networks (VANETs). Broadcasting of data and control packets is expected to be crucial in this environment. Both safety-related and non-safety applications rely on broadcasting for the exchange of data or status and advertisement messages. Most of the broadcasting traffic is designed to be delivered on a given frequency during the control channel ( $\mathrm{CCH})$ interval set by the WAVE draft standard. The rest of the time, vehicles switch over to one of available service channels (SCHs) for non-safety related data exchange. Although broadcasting in VANETs has been analytically studied, related works neither consider the WAVE channel switching nor its effects on the VANET performance. In this letter, a new analytical model is designed for evaluating the broadcasting performance on CCH in IEEE 802.11p/WAVE vehicular networks. This model explicitly accounts for the WAVE channel switching and computes packet delivery probability as a function of contention window size and number of vehicles.
\end{abstract}

Index Terms-802.11p, WAVE, broadcast, analytical model.

\section{INTRODUCTION}

$\mathbf{T}$ HE IEEE 802.11p task group [1] is working with the IEEE 1609 WAVE standard family [2] on a set of specifications to permit communications in the rapidly changing vehicular environment. The WAVE operating frequency is fixed in the DSRC (Dedicated Short Range Communication) band of 5.85-5.925 GHz. Within this range one $\mathrm{CCH}$ is reserved for system control and safety-related messages, and up to six SCHs are used to exchange non-safety data. WAVE [2] conceives an alternating access scheme: the channel time is divided into synchronization intervals with a fixed length of $100 \mathrm{~ms}$, consisting of equal-length alternating $\mathrm{CCH}$ and $\mathrm{SCH}$ intervals. During the $\mathrm{CCH}$ interval, all vehicular devices must tune on the $\mathrm{CCH}$ frequency for safety-related and system control data exchange; during the $\mathrm{SCH}$ interval vehicles (optionally) switch to one of the SCH frequencies. At the beginning of each interval, a $4 \mathrm{~ms}$-long guard time is set to account for radio switching delay and timing inaccuracies in the devices. Coordination between channels exploits a global time reference, the Coordinated Universal Time (UTC), provided by a global navigation satellite system.

Besides event-driven safety-related data, there are two types of messages that can be periodically transmitted over $\mathrm{CCH}$ : short status messages (beacons) and WBSS (WAVE-Basic

Manuscript received October 20, 2010. The associate editor coordinating the review of this letter and approving it for publication was F. A. Cruz-Perez.

C. Campolo and A. Molinaro are with the Department DIMET, Università Mediterranea di Reggio Calabria, Italy (e-mail: \{claudia.campolo, antonella.molinaro\}@unirc.it).

A. Vinel and Y. Koucheryavy are with the Department of Communication Engineering, Tampere University of Technology, Finland (e-mail: yk@cs.tut.fi, alexey.vinel@tut.fi).

Digital Object Identifier 10.1109/LCOMM.2011.122810.102007
Service Set) establishment and advertisement messages (WAVE Service Advertisements, WSAs). Beacons are used to periodically announce the presence of a vehicle to its neighbours; they carry status information about the vehicle position, its speed and direction. This data is useful to cooperative applications, such as collision avoidance, driver assistance, and cruise control. They require accurate and timely information from the context, hence typical beacon generation rates are in the range 5-10 Hz. WSAs are sent to advertise a WBSS set-up (and the related parameters) that provides connectivity and transport of non-safety services during the $\mathrm{SCH}$ interval.

Both WSAs and beacons are potentially beneficial to all vehicles in a given neighborhood, so they are transmitted as onehop broadcast by each sending node. Although broadcasting in VANETs has been studied from an analytical point of view [3]- [5], the related works have neither explicitly modelled the WAVE channel switching nor evaluated its effects on the system performance. In this work, broadcasting of beacons and WSAs has been analytically modelled by taking into account channel switching. The motivation to introduce a new analytical model designed for IEEE $802.11 \mathrm{p} /$ WAVE is twofold: (i) to investigate on capabilities and constraints of the upcoming standard; (ii) to help in designing mechanisms for improving the performance of broadcasting, whose inherent unreliability can be exacerbated by the envisioned WAVE channel switching.

\section{SySTEM Model}

Based on the IEEE 802.11p medium access control (MAC) specifications, a vehicular node is allowed to transmit only if it detects the channel idle for a time duration equal to AIFS (Arbitrary InterFrame Space) seconds ${ }^{1}$. Otherwise, the node waits for the channel to become free and then, it randomly selects a backoff value taken from a given range of integer numbers, called Contention Window $(W)$. This random value is used to set a backoff counter that is decremented at the end of each idle slot. As soon as the counter is decreased to zero, the node is allowed to access the channel. Broadcast frames are never acknowledged by the receivers.

In the proposed analytical model, each of $N$ vehicular nodes is supposed to have a packet - either beacon or WSA - ready to be transmitted at the beginning of every $\mathrm{CCH}$ interval. In agreement with the WAVE documents [2], the application layer is assumed to be aware of channel switching; this implies that packets are sent to the MAC layer only during the $\mathrm{CCH}$ interval. Moreover, according to [2], the wireless medium

\footnotetext{
${ }^{1}$ The IEEE 802.11p MAC has the same core mechanism of the Enhanced Distributed Channel Access (EDCA) specified in 802.11e. EIFS (Extended InterFrame Space) is used instead of AIFS whenever the physical layer indicates an unsuccessful transmission event.
} 


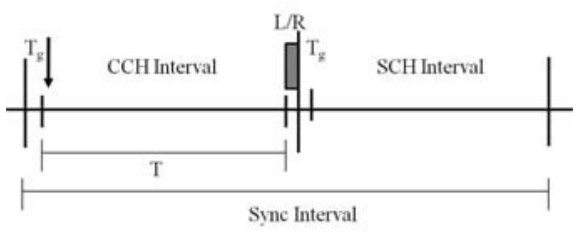

Fig. 1. Transmissions on the $\mathrm{CCH}$ interval.

is declared as busy during the guard interval to prevent multiple devices from attempting to transmit simultaneously upon switching, so all transmissions are delayed of a random backoff at the start of the $\mathrm{CCH}$ interval.

Due to the nature of beacon and WSA frames, it is reasonable to assume that their lifetime is bounded to one $\mathrm{CCH}$ interval; after this period non transmitted frames are dropped. Thereby, at the beginning of every $\mathrm{CCH}$ interval, a beacon or WSA frame is freshly generated and replaces the old one, in case it has not been transmitted yet. As a consequence, the MAC-layer buffer of a vehicular node keeps only one frame for $\mathrm{CCH}$ interval at a time. There is no need to model MAC queues for broadcast messages and there is no need to compute the frame delay. The following simplifying assumptions hold in this letter: (1) the radio channel is ideal, so bit errors are not considered; (2) all nodes are within reciprocal communication range (no hidden terminals); (3) the one-hop neighborhood of a node does not significantly change due to node mobility in the short time frame $(50 \mathrm{~ms})$ under analysis ${ }^{2}$; (4) the carrier sense range coincides with the communication range. The radio channel is assumed to be in one of three possible states (idle, success, collision); they respectively consider the cases of no transmission, one transmission and multiple simultaneous transmissions over the channel.

Let us define: $T_{s}$ as the duration of a successful transmission and $T_{c}$ as the collision duration. $T_{s}=T_{h}+L / R+A I F S$, where $L$ is the packet length, $R$ the data rate, and $T_{h}$ the duration of the PLCP (physical layer convergence protocol) preamble and header; $T_{c}=T_{h}+L / R+E I F S$. Since events occur on the $\mathrm{CCH}$ interval based on a discrete slotted time scale, the values $s=T_{s} / \sigma$ and $c=T_{c} / \sigma$ are the length measured in Slot Times $(\sigma)$ of a successful and a collided transmission, respectively. The useful duration $T$ of a $\mathrm{CCH}$ interval (with $T>W$ ), measured in Slot Times, is defined as $T=\left(T_{C C H}-T_{g}-L / R\right) / \sigma$, where $T_{C C H}$ is the duration of $\mathrm{CCH}$ interval and $T_{g}$ is the guard time, as depicted in Figure 1. Indeed, frames cannot be transmitted during the guard interval or if the residual time before the end of the $\mathrm{CCH}$ interval is shorter than the frame transmission time $L / R$, otherwise the transmission would be useless due to the mandatory channel switching.

The probability $P(l, n, w, k)(1 \leq l \leq w$ and $1 \leq k \leq n)$ is computed in Eq. (1) that $n$ vehicles are in the system that select backoffs from a contention window of $w$ slots, $(l-1)$ empty slots pass before the first transmission attempt, and $k$ vehicles transmit in the $l^{\text {th }}$ slot:

\footnotetext{
${ }^{2}$ Invariance of the one-hop neighbourhood should, more precisely, be required only during the packet transmission time; this is a less restrictive condition that makes the assumption even more reasonable.
}

$$
\begin{aligned}
P(l, n, w, k) & =\left(1-\frac{l-1}{w}\right)^{n} \\
& \cdot\left(\begin{array}{l}
n \\
k
\end{array}\right)\left(\frac{1}{w-l+1}\right)^{k}\left(1-\frac{1}{w-l+1}\right)^{n-k}
\end{aligned}
$$

The above probability is modeled through a Bernoulli process where $\frac{1}{w-l+1}$ is the probability of uniformly choosing any slot out of $(w-l+1)$ available slots.

Let us define $X(t, w, n)$ as the mean number of successful transmissions during the $\mathrm{CCH}$ interval, given that there are $t$ slots left in this interval, at most $w$ contention slots left at the vehicles backoff counter, when $n$ vehicles have not attempted to transmit yet:

$$
\begin{aligned}
& X(t, w, n)=\sum_{l=1}^{\min (w, t)}\{P(l, n, w, 1)[1+ \\
& +X(t-l+1-s, w-l, n-1)]+ \\
& \left.+\sum_{k=2}^{n} P(l, n, w, k) X(t-l+1-c, w-l, n-k)\right\}(2)
\end{aligned}
$$

The first two terms in Eq. (2) account for the probability that only one out of $n$ vehicles, choosing backoff from $w$ slots, successfully transmits in the $l^{\text {th }}$ slot, and $X(t-l+1-$ $s, w-l, n-1)$ is the mean number of successful transmissions in the remaining $(t-l+1-s)$ slots; while the third term models $k(k \geq 2)$ of $n$ vehicles transmitting in the $l^{\text {th }}$ slot and thus incurring collisions. Based on Eq. (1) and (2), and starting from $X(T, W, N)$, all possible numbers are recursively computed of first slots, among total $T$, which are empty, then the two cases (success and collision) followed by these empty slots are taken into account. Therefore, the probability of a successful packet delivery is computed as:

$$
\frac{X(T, W, N)}{N}
$$

With the given assumptions, a packet transmission could fail due to: (i) collisions with other nodes, whose backoff counters simultaneously decrease to zero; or (ii) packet dropping for expiry time due to a delayed backoff countdown that does not reach zero before the $\mathrm{CCH}$ interval end. This second case is unique to WAVE and its occurrence depends on $T_{\text {wait }}$, the maximum waiting time before the backoff counter becomes zero. In the worst case, $T_{\text {wait }}=(W-1) \cdot c$, each transmission attempt results in a collision, so that, at every time slot, a node finds the channel busy and freezes its backoff countdown for the collision duration $c$. On average, the time for the backoff counter to decrease to zero is significantly lower than $T_{\text {wait }}$, since some channel time could be occupied by successful transmissions $(s<c)$ or empty slots $(1)$. Moreover, $T_{\text {wait }}$ depends on the relationship between $N$ and $W$ as in Eq. (4):

$$
T_{\text {wait }}= \begin{cases}W-1 & \text { if } N=1 \\ s+W-2 & \text { if } N=2 \\ (N-1) \cdot s+[W-(N-1)-1] & \text { if } 2<N \leq W \\ \operatorname{floor}\left(\frac{N-W}{2}\right) \cdot c+\left[N-\operatorname{floor}\left(\frac{N-W}{2}\right)\right] \cdot s & \text { if } W<N<2 W+1 \\ (W-1) \cdot c & \text { if } N \geq 2 W+1\end{cases}
$$

where floor $(x)$ returns the closest integer less or equal than $x$. In particular, third line's condition holds if the difference between $c$ and $s$ is such that for any number of collisions 
TABLE I

MAC PARAMETERS

\begin{tabular}{lc}
\hline Parameter & Value \\
\hline Slot time $(\sigma)$ & $16 \mu \mathrm{s}$ \\
SIFS time & $32 \mu \mathrm{s}$ \\
AIFSN & 2 \\
EIFS & $188 \mu \mathrm{s}$ \\
Header duration $\left(\mathrm{T}_{h}\right)$ & $40 \mu \mathrm{s}$ \\
Packet generation rate & $10 \mathrm{~Hz}$ \\
\hline
\end{tabular}

TABLE II

PACKET LOSS PROBABILITY DUE TO CCH INTERVAL EXPIRY TIME

\begin{tabular}{l|cccccc}
\hline $\mathrm{N} \backslash W$ & 4 & 8 & 16 & 32 & 64 & 128 \\
\hline 10 & 0 & 0 & 0 & 0 & 0 & 0 \\
20 & 0 & 0 & 0 & 0 & 0 & 0 \\
30 & 0 & 0 & 0 & 0 & 0 & 0 \\
40 & 0 & 0 & 0 & 0 & 0 & 0.1 \\
50 & 0 & 0 & 0 & 0 & 0.1 & 0.26 \\
\hline
\end{tabular}

of multiplicity 2 denoted as $h\left(1 \leq h \leq f \operatorname{loor}\left(\frac{N-1}{2}\right)\right)$ this further condition is verified: $h \cdot c+(N-1-2 \cdot h) \cdot s+[W-$ $h-(N-1-2 \cdot h)-1]<(N-1) \cdot s+[W-(N-1)-1)]$.

If $T_{\text {wait }}<T$ then the channel time is long enough to accommodate a transmission attempt even in the worst case; so any vehicle can attempt to transmit once during the $\mathrm{CCH}$ interval. The impossibility for a node to transmit, and the consequent packet loss due to expiry time, instead, occurs only if $T_{\text {wait }} \geq T$. This condition reasonably holds with higher probability for long transmission times (low data rates or long packets), large $N$ and $W$.

\section{Model Validation and Numerical Results}

To validate the model, an event-driven custom simulation program has been developed in Matlab, that closely follows the $802.11 \mathrm{p}$ EDCA protocol specifications and the model assumptions. Results are shown for a set of parameter values that we consider as the most meaningful for analysis: the lowest $802.11 \mathrm{p}$ data rate -3 Mbps- is chosen to privilege robustness and reliability (very critical for unacknowledged broadcasting); 500-bytes long packets to account for additional security overhead, and a contention window size in the range (4-128). Other system parameters are listed in Table I.

Figure 2 (top) plots the successful packet delivery probability for different number of vehicles $N$ in the system, when varying the $W$ size. Analytical results (lines) closely match simulation results (symbols). The low values of packet delivery ratio, especially for high $N$ and low $W$ values, are due to the increase in collisions among nodes attempting to seize the channel at the beginning of the $\mathrm{CCH}$ interval. Figure 2 (bottom) shows that the overall packet loss probability monotonically decreases with the $W$ increase, whatever the number of nodes, thanks to better time diversity in transmission attempts. Although it is straightforward to infer that large windows reduce the collision probability, a large $W$, on its turn, is more prone to packet loss due to expiry time. Table II reports the fraction of packet loss only due to expiry time events. Under a wide range of operating conditions, packets
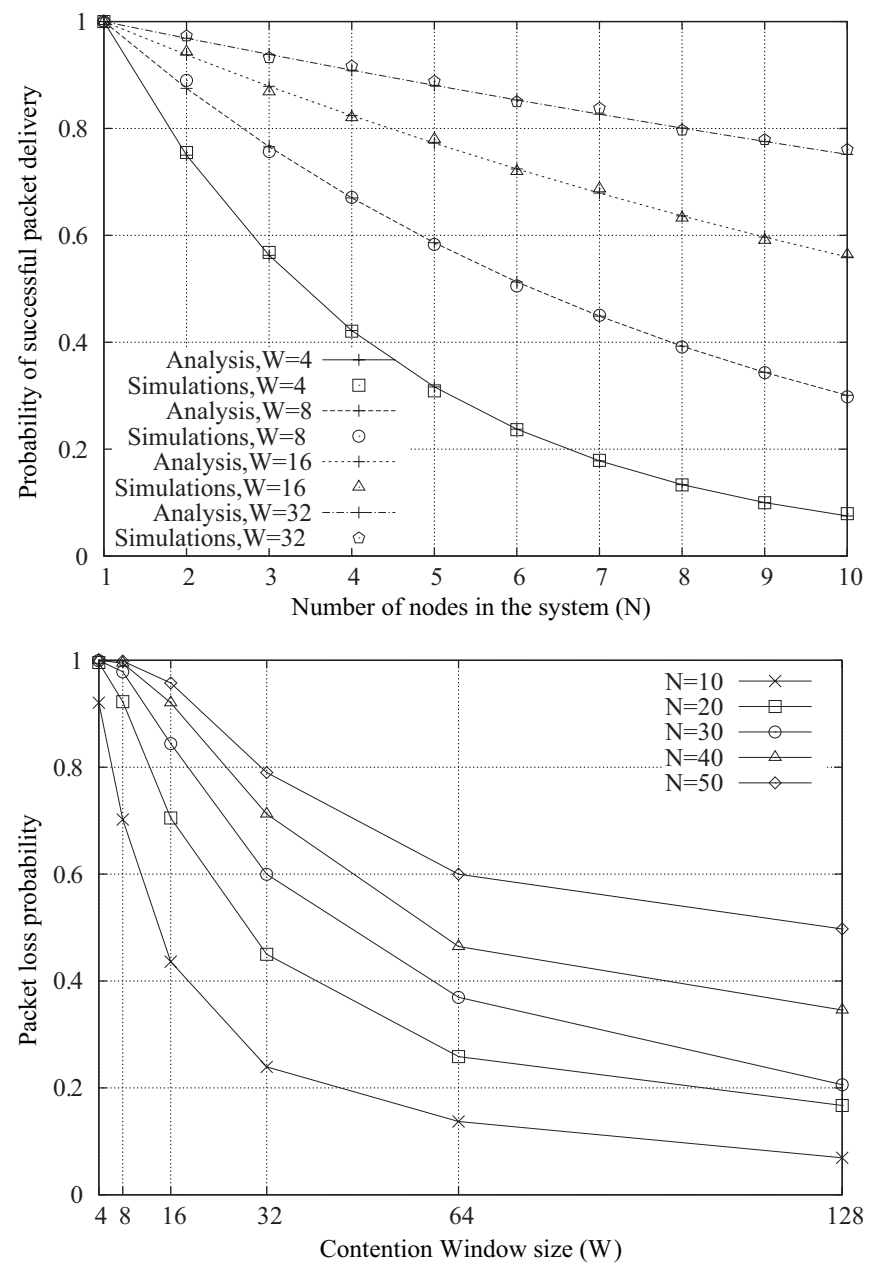

Fig. 2. Probability of successful broadcast delivery when varying $W$ (top), and packet loss probability for large $\mathrm{W}$ and high node densities (bottom).

are solely lost due to collisions; i.e., the $\mathrm{CCH}$ interval is long enough to accommodate one transmission attempt by all the nodes. This could not be the case when $N$ and $W$ increase.

Such findings call for the design of policies which better adapt the packet generation timing to the 802.11p/WAVE dynamics and for the definition of improved and more realistic analytics to carefully and precisely model them.

\section{REFERENCES}

[1] IEEE 802.11p/D10.0, Jan. 2010.

[2] "IEEE 1609.4/D6.0, Draft Standard for Wireless Accesses in Vehicular Environments (WAVE) - Multi-channel Operation, Mar. 2010.

[3] E. M. van Eenennaam, et al., "Exploring the solution space of beaconing in VANETs," in IEEE VNC 2009.

[4] A. Vinel, et al., "Estimation of a successful beacon reception probability in vehicular ad hoc networks," in IWCMC 2009.

[5] X. Ma and X. Chen, "Delay and broadcast reception rates of highway safety applications in vehicular ad hoc networks," in INFOCOM 2007. 